\title{
Identificação de prioridades em saúde: uma alternativa técnica de apoio à tomada de decisão
}

\author{
Identification of health priorities: a technical alternative \\ for support in decision-making
}

Marcelo Battesini ${ }^{1}$

Airton Fischmann ${ }^{1}$

Andreas Dittmar Weise ${ }^{2}$
${ }^{1}$ Departamento de Engenharia de Produção e Sistemas, Universidade Federal de Santa Maria. Av. Roraima, 1000, Camobi. 97.105-900 Santa Maria RS Brasil.

marcelo-battesini@ufsm.br

${ }^{2}$ Centro Estadual de

Vigilância em Saúde do RS.

\begin{abstract}
The scope of this study is to propose and discuss the application of an Index of Prioritization in Health (PIHealth). PIHealth is an objective criterion that reveals the magnitude of health problems and determinants in planning situations. PIHealth was used in identifying health priorities in different administrative health regions of the state of Rio Grande do Sul (RS) during the development of the 2009-2011 State Health Plan. The calculation of PIHealth made it possible to express the indicators analyzed within a given range ( 0 to 1) used to identify regional priorities (insufficient: 0 to 0.4 ; average: 0.41 to 0.69 ; and adequate: 0.7 to 1). The application of the index helped to identify the relationship in terms of magnitude of health problems based on an internal reference of the territories analyzed. Use of the index was a technical option in identifying priorities, which provided a simplification in the language used to express health problems and determinants. Its discretionary power and the intuitive simplicity of its interpretation showed that PIHealth is a promising tool, providing objective answers to key questions frequently addressed in health planning contexts.
\end{abstract}

Key words Priorities in Health, Decision support techniques, Health planning, Health management, Health indicators
Resumo Oobjetivo deste estudo épropor e discutir a aplicação de um Índice de Priorização em Saúde $\left(I P_{\text {Saúde }}\right)$. OIP $P_{\text {Saúde }}$ é um critério objetivo que evidencia a magnitude de problemas e determinantes de saúde em situações de planejamento. O IP $P_{\text {Saúde }}$ foi utilizado na identificação de prioridades de saúde nas diferentes nas regiões de administrativas do Rio Grande do Sul (RS), durante o desenvolvimento do Plano Estadual de Saúde 2009-2011. O cálculo do IP ${ }_{\text {Saúde }}$ permitiu expressar os indicadores analisados em um mesmo intervalo (0 a 1) utilizado na identificação de prioridades regionais (insuficiente, 0 a 0,4; médio, 0,41 e 0,69; e adequado, 0,7 a 1). A aplicação do Índice auxiliou na identificação da magnitude relacional de problemas de saúde com base em uma referência interna aos territórios analisados. $O$ emprego do indice representou uma opção técnica na identificação de prioridades que proporcionou uma simplificação na linguagem utilizada para expressar problemas e determinantes de saúde. Sua capacidade discricionária e a singeleza intuitiva de sua leitura demonstraram que o IP Saúde é uma ferramenta promissora, ao proporcionar respostas objetivas a questões frequentemente postas em contextos de planejamento em saúde.

Palavras-chave Prioridades em saúde, Técnicas de apoio para a decisão, Planejamento em saúde, Gestão em saúde, Indicadores de saúde 


\section{Introdução}

A tomada de decisão é uma prerrogativa de atores implicados em um processo de gestão, que demanda a definição de prioridades como consequência natural do desequilíbrio entre necessidades e recursos.

Planejar, organizar, controlar e avaliar ações e serviços de saúde, presentes em seu território, são funções administrativas legalmente preconizadas aos gestores do Sistema Único de Saúde (SUS) pelas Leis 8.080/901 e 8.142/902. Competências reiteradas no âmbito do Pacto pela Saúde, com a proposição de um sistema de planejamento do SUS, dado na Portaria 3085/06/MS 3 , na busca do desenvolvimento da função planejamento e de um alinhamento dos instrumentos utilizados no âmbito do SUS: o plano de saúde, a programação anual e o relatório de gestão. Iniciativas essas mais recentemente revitalizadas e estimuladas pelo Decreto $7.508 / 11^{4}$, ao propor uma articulação interfederativa baseada em um processo de planejamento ascendente e integrado a partir do nível local.

O esforço para a qualificação do planejamento parece justificável frente à realidade observada, que admite afirmações preocupantes: tradicionalmente os diagnósticos de saúde presentes em planos de saúde não guardam muita relação com as propostas de ação, seja por insuficiência de análise ou inércia do sistema de prestação de serviços ${ }^{5}$; o planejamento no SUS é ritualístico e sem compromissos com a solução de problemas ${ }^{6}$; e a inserção de ações de planejamento nas instituições públicas de saúde tem sido precária, e muitas vezes, orientada pela produção burocrática de um plano documento e de demonstrações físicas da implantação de unidades de saúde ${ }^{7}$.

Apesar das diferentes formas de planejamento no SUS não apresentarem homogeneidade doutrinária no plano acadêmico, parece não haver dúvidas quanto ao papel central do estado enquanto agente disparador e coordenador, nem tampouco da necessidade de consolidar espaços de poder compartido, apesar do debate em torno da forma como se obter consensos neste processo ${ }^{7-9}$.

A relação entre atores vem se alterando ao longo do tempo na busca de uma representatividade inclusiva entre os sujeitos presentes no processo decisório, como forma de melhorar a função planejamento. Aos profissionais de planejamento em saúde cumpre o desafio de desenvolver estratégias para ampliar a capacidade de diferentes atores reconhecerem problemas de saúde prioritários, de modo a incrementar a consis- tência entre as ações planejadas e as necessidades de saúde da população.

A apreensão do conjunto de informações resultante de análises da situação de saúde é capaz de desafiar os diferentes atores partícipes do planejamento em saúde, usualmente representantes da comunidade, gestores e profissionais de saúde. A necessidade de abstrair significado de conjuntos de indicadores expressos por valores numéricos, razões, taxas e coeficientes, normalmente caracterizados em unidades de medida diversas, introduz grande dificuldade à compreensão de um quadro global da situação de saúde. Mesmo técnicos experientes, dificilmente dominam todos os indicadores e dimensões que compõem um diagnóstico da situação de saúde, tendendo a limitar o alcance de suas análises às temáticas $\mathrm{e}$ programas com as quais trabalham.

Cabe ao nível técnico propor uma simplificação inclusiva na linguagem utilizada para expressar a situação de saúde, de modo a facilitar sua compreensão por não especialistas, ampliando as possibilidades de consenso e permitindo responder questões relevantes e indutoras no direcionamento da aplicação de recursos em saúde. Quais são os problemas de saúde prioritários em um dado território? Em que territórios os investimentos devem ser priorizados?

De modo a lidar com estas questões, este trabalho tem como objetivo propor um Índice de Priorização em Saúde ( $\left(\mathrm{IP}_{\text {Saúde }}\right)$ e discutir sua aplicação, enquanto alternativa técnica de apoio à tomada de decisão em situações de planejamento em saúde. A seguir são apresentadas as seções métodos, os aspectos conceituais e a aplicação, a discussão e as perspectivas e considerações finais.

\section{Métodos}

A proposta do IP ${ }_{\text {Saúde }}$ é resultado de reflexão teórica frente a uma problemática estabelecida no mundo real, o que caracteriza um propósito de pesquisa aplicada, com objetivo de natureza analítica, que utiliza a pesquisa bibliográfica e o levantamento de campo como fonte de dados e procedimento de coleta ${ }^{10,11}$.

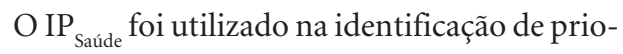
ridades de saúde nas diferentes nas regiões administrativas de saúde do Rio Grande do Sul (RS). O índice foi aplicado na análise da situação de saúde, durante desenvolvimento do Plano Estadual de Saúde (PS-RS), para o período 2009-2011.

A elaboração do PS-RS se valeu do conhecimento dos servidores da Secretaria Estadual de 
Saúde (SES-RS), o que caracterizou um avanço em relação à forma anteriormente utilizada, conduzida com apoio de uma consultoria externa. Essa opção levou a criação de um grupo de trabalho específico composto por técnicos dos diferentes departamentos, coordenados pela Assessoria Técnica de Planejamento da SES-RS.

Inicialmente foi realizado um alinhamento entre o Plano Plurianual (anteriormente aprovado), o Termo de Compromisso de Gestão Estadual e as diretrizes do Pacto pela Saúde, identificando as demandas e os limitantes neles estabelecidos. Após, foi realizado um diagnóstico da situação, que se seguiu à etapa de proposição de ações de saúde frente aos problemas prioritários identificados. Por fim, o texto base foi revisado e submetido ao Conselho Estadual de Saúde (CES) e uma vez aprovado o plano foi publicado.

Durante a etapa de diagnóstico, os programas e as áreas técnicas da SES-RS produziram estudos longitudinais para as regiões administrativas de saúde, em relação a diferentes problemas, com base em indicadores de morbimortalidade da população.

A situação de saúde das Coordenadorias Regionais de Saúde (CRS) também foi caracterizada com base no cálculo do $\mathrm{IP}_{\text {Saúde }}$ para um conjunto de setenta e seis (76) indicadores utilizados pela Rede de Análise de Dados e Indicadores de Saúde (READIS), incluindo indicadores Demográficos, Socioeconômicos e de Mortalidade, Morbidade, Fatores risco, Recursos e Cobertura, referentes ao ano de 2008. A READIS acompanha um total de cento e cinco (105) indicadores, historicamente usados para análises de situação, tendo como base os utilizados na Rede Interagencial de Informações para a Saúde (RIPSA). De modo a facilitar sua visualização pelos diferentes atores, essas análises foram expressas em mapas com auxílio do Tabwin regionalizados com base nas dezenove (19) Coordenadorias Regionais de Saúde (CRS), regiões administrativas da SES (RS) utilizadas como unidade territorial de análise.

A análise desses estudos foi realizada por grupos de trabalho, compostos por técnicos das CRS, organizados por macrorregiões de saúde, na busca de uma leitura regionalizada da situação de saúde no RS. As prioridades apontadas com base no $\mathrm{IP}_{\text {Saúde }}$ instrumentalizaram as discussões e a proposição de ações para o enfrentamento dos problemas subsidiando a tomada de decisão pelos gestores regionais e estaduais em relação à alocação de recursos, assim como a análise do plano pelos conselheiros de saúde, quando da sua aprovação junto ao CES.
O resultado deste esforço foi apresentado na forma de um capítulo do PS-RS - 2009-2011, onde foram caracterizadas as especificidades e prioridades em termos dos valores de $\mathrm{IP}_{\text {Saúde }}$ e de regiões administrativas de saúde.

\section{Índice de Priorização em Saúde: aspectos conceituais e aplicação}

O Índice de priorização em Saúde $\left(\mathrm{IP}_{\text {Saúde }}\right)$ se caracteriza como uma alternativa técnica para a identificação de prioridades em processos de tomada de decisão. A ideia central subjacente ao $\mathrm{IP}_{\text {Saúde }}$ é simplificar e uniformizar a forma de expressar indicadores utilizados na representação da situação de saúde, de modo a facilitar sua compreensão pelos atores envolvidos no planejamento e possibilitar a apreciação simultânea de um conjunto maior de dimensões. A determinação do IP Saúde $_{\text {demanda: }}$

- a definição de territórios sobre os quais existe capacidade de ação e responsabilidade, que delimitam o nível de análise adotado, a exemplo de distritos (municipal), regiões de saúde (estadual) ou estados (nacional);

. a escolha de indicadores relevantes a serem considerados no diagnóstico de situação, que devem ser capazes de representar os problemas e os determinantes de saúde objeto de priorização; e,

. o estabelecimento de intervalos de priorização, que correspondem aos limites de referência a serem utilizados na análise.

O cálculo do $\mathrm{IP}_{\text {Saúde }}$ independe da forma como foram eleitos os territórios ou regiões, assumindo-os como ${ }^{12}$ o resultado de uma produção coletiva com materialidade histórica, social e configuração espacial singular, compatível com a organização político-administrativa e institucional.

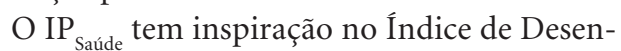
volvimento Humano (IDH), utilizando a mesma forma de cálculo de seus índices de dimensão. O IDH é calculado a partir da média aritmética de três índices, que procuram representar dimensões básicas do desenvolvimento humano: (i) vida longa e saudável, medida pela esperança de vida ao nascer; (ii) conhecimento, medido pela taxa de analfabetismo de adultos e pela taxa de escolarização bruta combinada (ensino primário, secundário e superior); e (iii) nível de vida digno, medido pelo Produto Interno Bruto (PIB) per capita em dólares ${ }^{13}$.

$$
\begin{gathered}
\mathrm{IP}_{\text {Saúde }}=\frac{\text { Valor observado }- \text { Valor Mínimo }}{\text { Valor Máximo - Valor Mnimo }} \\
\text { Equação 1 }
\end{gathered}
$$


Os valores assim obtidos expressam a magnitude dos problemas de saúde em um dado território (valor observado) em relação aos demais considerados (valores mínimo e máximo). A Equação 1 fornece os valores de $\mathrm{IP}_{\text {Saúde }}$ para indicadores do tipo maior é melhor (quanto maior o valor do indicador melhor o estado de saúde da população), já para indicadores do tipo menor é melhor, seu valor é calculado subtraindo da unidade o resultado obtido.

Em ambos os casos, o valor do $\mathrm{IP}_{\text {Saúde }}$ é expresso em termos de um intervalo que varia sempre entre zero e um ( 0 a 1 ), o que permite a uniformização direcional na sua leitura. Desse modo, valores de $\mathrm{IP}_{\text {Saúde }}$ próximos à zero sempre representam prioridades em saúde e, inversamente, valores próximos à unidade representam um desempenho de referência, uma performance a ser alcançada pelos demais territórios analisados.

Essa padronização no intervalo de variação e uniformização de leitura permitiu estabelecer três intervalos de priorização convergentes entre os diferentes indicadores objeto de análise, que funcionam como um critério técnico unificado para a identificação das prioridades, onde valores de $\mathrm{IP}_{\text {Saúde }}$ entre:

.0 e 0,4 indicam desempenho relativo insuficiente e uma prioridade em saúde;

. 0,41 e 0,69 representam um desempenho relativo médio a ser melhorado; e

. 0,7 a 1 expressam um desempenho relativo adequado e uma referência a ser alcançada pelos demais territórios.

A escolha dos intervalos foi intencional e intuitiva: territórios com valores de $\mathrm{IP}_{\text {Saúde }}$ igual ou superior a 0,7 naturalmente se sobressaem em relação aos demais e, similarmente, territórios com valores igual ou inferior a 0,4 apresentam resultado relacional insuficiente quando considerado o conjunto de territórios analisados.

A Figura 1 expressa visualmente os intervalos de priorização e também o significado dos valores do IP $_{\text {Saúde }}$, enquanto uma medida de distância relativa entre o valor observado, o valor mínimo e o valor máximo. Com o objetivo de facilitar a análise de prioridades e a representação espacial territorializada do $\mathrm{IP}_{\text {Saúde }}$, cada um dos três intervalos foi expresso com as três cores utilizadas em semáforos: cor vermelha ( $\left.\operatorname{IP}_{\text {Saúde }} \leq 0,4\right)$, amarela $\left(0,41 \geq \operatorname{IP}_{\text {Saúde }} \leq 0,69\right)$ e verde $\left(\mathrm{IP}_{\text {Saúde }} \geq 0,7\right)$.

Uma forma simplificada de descrever o cál-

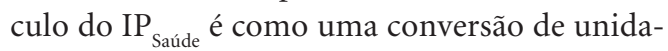
de, onde as medidas originais dos indicadores são expressas em termos de um mesmo intervalo de variação, utilizado como critério unificado e unidirecional na apreciação de um conjunto de indicadores. Essa característica e a influência do IDH na sua forma de cálculo definiram sua denominação enquanto um índice. Entendido como índice de referência que expressa a situação mais recente do problema e sua respectiva data de apuração $0^{14}$, podendo assumir formas ${ }^{15}$ simples, a exemplo de um valor observado em um dado período expresso como uma percentagem em relação a um período base, ou formas mais complexas, como um índice de preços ao consumidor.

A opção metodológica de calcular o IP Saúde' , individualmente, para cada indicador tem o objetivo de expressar a especificidade dos problemas e determinantes nos diferentes territórios, o que representa uma escolha pela facilidade de compreensão e de utilização no processo de tomada de decisão, inclusive por não especialistas, apesar de ser possível criar um indicador composto ou sintético, a partir da utilização do IP $_{\text {Saúde }}$ de diferentes indicadores.

São exemplos de índices sintéticos o próprio IDH, o Îndice de Necessidades em Saúde, desenvolvido pela Coordenação de Epidemiologia do município de São Paulo para identificar áreas e grupos populacionais a serem priorizados na oferta de serviços de saúde, e seu homônimo, elaborado pela Fundação João Pinheiro para medir a desigualdade entre municípios e citado no Plano de Saúde do Estado de Minas Gerais 2008-2011. Além do Îndice de Desempenho do SUS (IDSUS) proposto em 2011 pelo Ministério da Saúde para aferir o desempenho do Sistema de Único de Saúde (SUS) quanto ao acesso obtido e à efetividade de diferentes níveis de atenção.

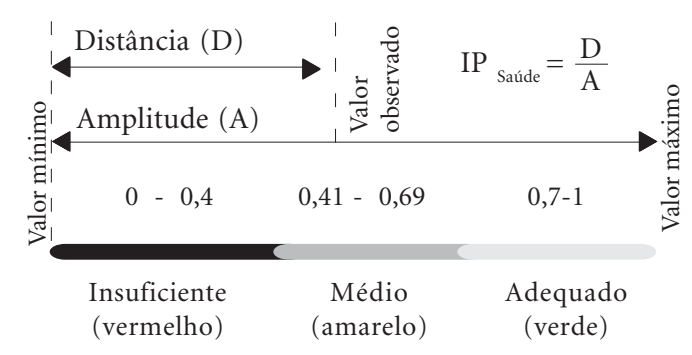

Figura 1. Intervalos de priorização e significado do IP Saúde . 
A importância da aplicação do $\mathrm{IP}_{\text {Saúde }}$ na priorização de problemas de saúde, caracterizados com base em indicadores, pode ser visualizada a partir de sua aplicação em uma situação de planejamento em saúde. A seguir são apresentados o cálculo e as análises do $\mathrm{IP}_{\text {Saúde }}$ para três dos indicadores adotados na caracterização da situação de saúde do PES-RS, para o período entre 2009-2011.

A Tabela 1 apresenta os valores do $\mathrm{IP}_{\text {Saúde }}$ para a $1^{\mathrm{a}}, 11^{\mathrm{a}}$ e $16^{\mathrm{a}} \mathrm{CRS}$, calculados com base na Equação 1, para os indicadores Coeficiente de Mortalidade Infantil $\left(\mathrm{CM}_{\text {Infantil }}\right.$ número de óbitos em crianças com menos de um ano por 1000 nascidos vivos, ano base 2007), Expectativa de Vida ao nascer $\left(\mathrm{EV}_{\text {Nascer }}\right.$, em anos de vida, considerando ambos os sexos, período de 2005 a 2007) e Cobertura Vacinal contra a Poliomielite $\left(\mathrm{CV}_{\text {Pólio, }}\right.$, $\%$ de crianças vacinadas contra pólio oral em relação à população de crianças com idade inferior a um ano, ano base 2007).

A capacidade discricionária conferida pelo uso do IP $_{\text {Saúde }}$ pode ser observada em relação ao $\mathrm{CM}_{\text {Infantil }}$ que se apresenta insuficiente na $11^{\text {a }} \mathrm{CRS}$ $\left(\mathrm{IP}_{\text {Saúde }}=0,33\right)$ por apresentar valor muito distante daquele obtido pela $16^{\text {a }}$ CRS ( IP $_{\text {Saúde }}=1$ ). Situação similar pode ser observada em relação à $\mathrm{EV}_{\text {Nascer }}$ na $1^{\text {a }} \mathrm{CRS}$ (72,6 anos), que apesar de possuir valor supostamente elevado quando observado na unidade original, se apresenta insuficiente quando comparado àqueles obtidos pelas demais regiões administrativas do RS.

Uma forma interessante de observar indicadores prioritários é a sua representação espacial territórializada. A Figura 2 apresenta os valores de $\mathrm{IP}_{\text {Saúde }}$ para as dezenove (19) regiões adminis- trativas do RS, gerados no Tabwin, em relação aos os indicadores $\mathrm{CM}_{\text {Infantil }} \mathrm{CV}_{\text {Pólio }} \mathrm{e} \mathrm{EV}_{\text {Nascer }}$, onde a $1^{\text {a }}, 11^{\text {a }}$ e $16^{\text {a }}$ CRS aparecem destacadas com círculos tracejados.

A simplicidade da leitura visual de problemas e determinantes de saúde prioritários e a possibilidade de apreciação da distribuição de prioridades foram destacadas pelos técnicos dos grupos de trabalho responsáveis por desenvolver uma análise regionalizada da situação de saúde no RS. Essas características reforçam a ideia cen-

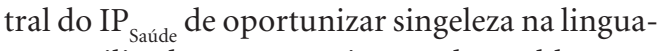
gem utilizada na comunicação de problemas e determinantes de saúde aos atores partícipes do processo de planejamento.

Além dos mapas foi conduzida uma análise para identificar as CRS prioritárias, assim considerados por apresentarem desempenho relacional insuficiente para um conjunto expressivo de indicadores, em termos do IP ${ }_{\text {Saúde }}$. A Tabela 2 apresenta os percentuais de indicadores para cada um dos três intervalos de priorização por CRS, considerando o conjunto de indicadores (76) analisados durante o desenvolvimento do PES-RS.

A caracterização de CRS apresentando um grande número de indicadores com valor de IPSaúde insuficiente, a exemplo da $3^{\mathrm{a}}$, 7a, $8^{\mathrm{a}}$ e $10^{\mathrm{a}} \mathrm{CRS}$, coincidiu com as regiões historicamente identificadas como as de pior desempenho em termos de indicadores de saúde, representando uma considerável parcela da população do RS.

A capacidade discricionária dos intervalos adotados foi constatada com base em uma análise de correlação de Pearson, que indicou uma forte relação inversa $(-0,679)$ entre a primeira coluna $\left(\mathrm{IP}_{\text {Saúde }} \leq 0,4\right)$ e a terceira $\left(\operatorname{IP}_{\text {Saúde }} \geq 0,7\right)$, ou

Tabela 1. Valores do $\mathrm{IP}_{\text {Saúde }}$ para indicadores e CRS selecionados.

\begin{tabular}{|c|c|c|c|c|c|c|}
\hline Indicador & CRS & Valor observado & Valor Mínimo & Valor Máximo ${ }^{(1)}$ & & \\
\hline \multirow[t]{3}{*}{$\mathrm{CM}_{\text {Infantil }}$} & $1^{\mathrm{a}}$ & 12,4 óbitos & 9,6 óbitos & 19,9 óbitos & 0,73 & Adequado \\
\hline & $11^{\mathrm{a}}$ & 16,5 óbitos & & & 0,33 & Insuficiente \\
\hline & $16^{\mathrm{a}}$ & 9,6 óbitos & & & $1,00^{(2)}$ & Adequado \\
\hline \multirow{3}{*}{$\mathrm{EV}_{\text {Nascer }}$} & $1^{\mathrm{a}}$ & 72,6 anos & 72,4 anos & 75,6 anos & 0,06 & Insuficiente \\
\hline & $11^{\mathrm{a}}$ & 75,6 anos & & & $1,00^{(3)}$ & Adequado \\
\hline & $16^{\mathrm{a}}$ & 75,2 anos & & & 0,88 & Adequado \\
\hline \multirow[t]{3}{*}{$\mathrm{CV}_{\text {Pólio }}$} & $1^{\mathrm{a}}$ & $90,4 \%$ & $89,7 \%$ & $106 \%$ & 0,04 & Insuficiente \\
\hline & $11^{\mathrm{a}}$ & $89,7 \%$ & & & 0,00 & Insuficiente \\
\hline & $16^{\mathrm{a}}$ & $90,6 \%$ & & & 0,06 & Insuficiente \\
\hline
\end{tabular}

(1) Valor Mínimo e Máximo considerando o conjunto das 19 CRS. ${ }^{(2)} \operatorname{IP}_{\text {Saúde }}$ para $\mathrm{CM}_{\text {Infantil }}{ }^{1{ }^{a} C R S}=1-(9,6-9,6) /(19,9-9,6)=1 .{ }^{(3)}$ $\mathrm{IP}_{\text {Saúde }}$ para $\mathrm{EV}_{\text {Nascer }}{ }^{11^{\mathrm{a}} \mathrm{CRS}}=(75,6-72,4) /(75,6-72,4)=1$ 

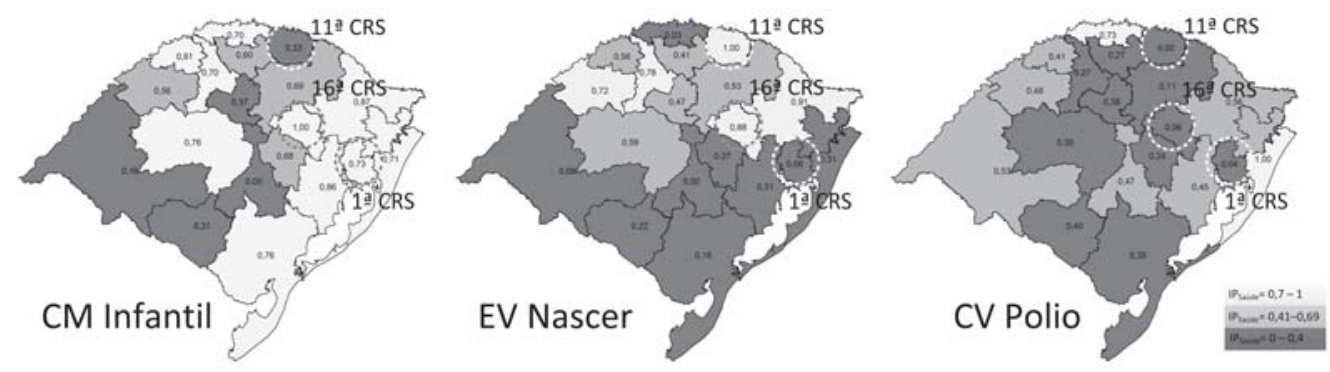

Figura 2. Valores de $\mathrm{IP}_{\text {saúde }}$ para as 19 regiões administrativas do RS.

Tabela 2. Percentual de indicadores por intervalo de priorização por CRS e população.

\begin{tabular}{ccccc}
\hline CRS & $\mathbf{0 , 4}$ (insuficiente) & $\mathbf{0 , 4 1}$ a $\mathbf{0 , 6 9}$ (médio) & $\mathbf{2 0 , 7}$ (adequado) & População 2009 \\
\hline $1^{\mathrm{a}}$ & 39 & 24 & 37 & 3.639 .964 \\
$2^{\mathrm{a}}$ & 28 & 30 & 42 & 766.783 \\
$3^{\mathrm{a}}$ & 46 & 32 & 22 & 871.036 \\
$4^{\mathrm{a}}$ & 26 & 34 & 40 & 578.197 \\
$5^{\mathrm{a}}$ & 17 & 25 & 58 & 1.065 .782 \\
$6^{\mathrm{a}}$ & 26 & 46 & 28 & 604.981 \\
$7^{\mathrm{a}}$ & 45 & 17 & 38 & 182.282 \\
$8^{\mathrm{a}}$ & 42 & 20 & 38 & 204.916 \\
$9^{\mathrm{a}}$ & 28 & 38 & 34 & 133.618 \\
$10^{\mathrm{a}}$ & 47 & 29 & 24 & 531.106 \\
$11^{\mathrm{a}}$ & 28 & 23 & 49 & 221.091 \\
$12^{\mathrm{a}}$ & 20 & 33 & 47 & 232.924 \\
$13^{\mathrm{a}}$ & 29 & 41 & 30 & 335.771 \\
$14^{\mathrm{a}}$ & 26 & 23 & 51 & 228.632 \\
$15^{\mathrm{a}}$ & 38 & 24 & 38 & 165.220 \\
$16^{\mathrm{a}}$ & 24 & 26 & 50 & 371.115 \\
$17^{\mathrm{a}}$ & 22 & 32 & 46 & 227.020 \\
$18^{\mathrm{a}}$ & 35,5 & 29 & 35,5 & 343.829 \\
$19^{\mathrm{a}}$ & 38 & 21 & 41 & 209.861 \\
\hline
\end{tabular}

seja, na maioria das CRS um alto percentual indicadores com valor insuficiente corresponde a um baixo percentual de indicadores com valor adequado, e vice versa.

\section{Discussão e perspectivas de aplicação do IP Saúde $_{\text {}}$}

A tomada de decisão em relação à prioridade de problemas e estratégias de ação em saúde demanda legitimidade dos sujeitos implicados, tipicamente representantes da comunidade, técnicos e gestores.
Os atores envolvidos podem definir prioridades com base em critérios objetivos ou em preferencias subjetivas ${ }^{5}$. Entre os critérios objetivos clássicos na identificação de problemas e determinantes de saúde propostos, pautados por uma racionalidade epidemiológica, estão a magnitude, o potencial de disseminação, a relevância, a severidade e a vulnerabilidade.

A definição à priori dá transparência à forma de alocação dos recursos explicitando o uso dos mesmos princípios para todos os indivíduos, evitando que as decisões sejam discricionárias, ficando a critério de quem detém o poder decidir caso a caso. 
O mau uso do poder discricionário associado à insuficiente participação da comunidade na definição de prioridades autoriza a falta de objetividade na tomada de decisão, prática usual na gestão de recursos na área da saúde ${ }^{16}$, a exemplo da utilização de critérios de decisão variados, flutuantes e dependentes de disposições pessoais, dos obstáculos que tal decisão poderia enfrentar para ser ratificada, ou do percurso identificado para sua implementação; da utilização de decisões não explicitamente voltadas a resultados ou ao impacto sobre a situação de saúde da população, "sujeito oculto" do discurso organizacional; da permeabilidade a pressões político-partidárias na escolha de gerentes e diretores; da decisão em função da oportunidade de obtenção de recurso, cuja finalidade possui baixa sintonia em relação a objetivos previamente estabelecidos; além da utilização de critérios subjetivos, tais como, a humanidade, a intuição e a disposição pessoal.

A justa priorização de recursos deve ser explícita, sobretudo quando estes forem escassos ou limitados, obrigando a realização de escolhas éticas ao definir a quem e quais os cuidados de saúde serão oferecidos ${ }^{17}$. A ausência de um processo prévio e participativo de priorização tende a ampliar o espaço para uso do poder discricionário pelos gestores, sujeito a adoção de uma racionalidade política em um contexto organizacional, que admite a tomada de decisão burocrática com base em fatores subjetivos e na diversidade de interesses dos atores.

A proposição do $\mathrm{IP}_{\text {Saúde }}$ simplifica a forma de representar os indicadores, o que pode ampliar a capacidade de participação de não especialistas nos processos de tomada de decisão em saúde, correspondendo à adoção de uma postura de acolhimento aos diferentes atores.

Em sua aplicação durante desenvolvimento PS-RS, para o período 2009-2011, o índice serviu de ponto de partida para as discussões dos grupos técnicos responsáveis pela identificação de problemas e regiões de saúde prioritárias, tendo sido utilizado como critério objetivo para evidenciar a magnitude relacional de problemas e determinantes de saúde. É importante destacar que a utilização do $\mathrm{IP}_{\text {Saúde }}$, não excluiu o uso concomitante de critérios qualitativos, tanto políticos quanto técnicos, no desenvolvimento dos diagnósticos regionais, assim como não exclui a utilização do repertório cognitivo dos atores na apreensão da situação de saúde ${ }^{18}$.

A uniformização direcional no raciocínio adotado para a análise dos indicadores simplificou e facilitou sua interpretação. Essa caracterís- tica foi especialmente útil na apresentação na proposta de plano junto ao Conselho de saúde, ao permitir a uniformização da lógica de análise, ou seja, valores altos de $\mathrm{IP}_{\text {Saúde }}$ sempre correspondem a um desempenho adequado para todos os indicadores considerados.

A utilização de intervalos de priorização idênticos (insuficiente $\mathrm{IP}_{\text {Saúde }} \leq 0,4$ e adequado $\mathrm{IP}_{\text {Saúde }}$ $\geq 0,7)$ para todos indicadores facilitou a apreciação simultânea de um conjunto maior de indicadores, possibilitando a construção cognitiva de um panorama ampliado da situação de saúde. Essa propriedade auxiliou a atenuar disputas técnicas entre diferentes programas que, normalmente, tendem a defender vaidosamente seus indicadores como prioritários. A capacidade de compatibilizar diferentes perspectivas se mostrou uma grande qualidade do $\mathrm{IP}_{\text {Saúde }}$, ao permitir o reconhecimento de prioridades pelos diferentes pares mesmo sem comungarem o mesmo domínio das distintas áreas.

As escolhas do nível e da referência de análise não são imparciais, uma vez que a definição do nível de análise guarda relação direta com a legitimidade e possibilidade de ação (localidade, distrito, município, região de saúde, estado ou país), enquanto a referência de análise está relacionada ao grau de tolerância/rigor desejado na identificação de problemas prioritários (valor de referencia a ser alcançado). A adoção de uma referência interna aos territórios analisados representa uma opção técnica que busca a adoção de valores oportunos e adequados às necessidades da população, minimizando o uso de valores de referência que atendam apenas às politicamente convenientes.

A importância da utilização de uma referência de análise interna está relacionada à imparcialidade do julgamento produzido, o que pode ser observado em relação ao indicador $\mathrm{CM}_{\text {Infantil }}$ cujo desempenho da $11^{\text {a }} \mathrm{CRS}$ foi insuficiente $\left(\mathrm{IP}_{\text {Saú- }}\right.$ $\left.{ }_{\text {de }}=0,33\right)$, sinalizando uma prioridade em saúde no âmbito do RS, apesar de expressar uma taxa de mortalidade de 16,5 óbitos por mil nascidos vivos, taxa que pode ser considerada aceitável, ou até mesmo uma meta a ser alcançada, em alguns Estados brasileiros.

A conversão em proposições de ações de saúde consistentes com as prioridades identificadas parece ser dependente do amadurecimento democrático do processo de planejamento e de um maior equilíbrio na distribuição do poder entre gestores e representantes da comunidade.

A reversão dessa situação também depende de um posicionamento de profissionais de pla- 
nejamento em saúde, uma vez que o funcionamento do Conselho de Saúde (CMS) pode não garantir o efetivo controle pela comunidade ${ }^{19,20}$, podendo resultar em uma prática burocratizada como consequência da relação assimétrica de poder entre os atores. A autodeterminação e a conquista efetiva da democracia e da participação da comunidade demanda conhecimento emancipador capaz de instrumentalizar a participação e o posicionamento em processos de tomada de decisão ${ }^{21}$.

De um modo geral, a aplicação do $\mathrm{IP}_{\text {Saúde }}$ se apresentou como uma ferramenta útil e promissora ao proporcionar respostas objetivas a questões essenciais a serem respondidas em situações de proposição de estratégias de ação sobre problemas de saúde e territórios.

\section{Conclusão}

O recente esforço para consolidar a função planejamento no âmbito do SUS, conjugado à necessidade de ampliar a participação de atores em situações de tomada de decisão, estabelece um desafio aos profissionais de planejamento em saúde. Contexto esse, propício à introdução de ferramentas de apoio a tomada de decisão em relação à aplicação de recursos públicos.

A proposta do $\mathrm{IP}_{\text {Saúde }}$ busca preencher essa lacuna reforçando a necessidade de acolher não especialistas em contextos de planejamento, através da simplificação da linguagem utilizada para expressar problemas e determinantes de saúde. O IP ${ }_{\text {Saúde }}$ foi utilizado como critério objetivo de apoio a tomada de decisão, tendo auxiliado na identificação da magnitude relacional de problemas de saúde pela uniformização do raciocínio adotado na análise de situação de saúde e pela utilização de uma referência interna aos territórios analisados. A aplicação do $\mathrm{IP}_{\text {Saúde }}$ possibilitou a identificação de indicadores e territórios prioritários, no desenvolvimento do Plano de Saúde do Rio Grande do Sul (PS-RS) para o período 2009-2011, o que ressaltou sua capacidade discricionária a singeleza intuitiva de sua leitura.

Espera-se que o resultado obtido com a apli-

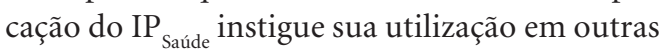
situações e contextos, especialmente naqueles que estimulem a presença de não especialistas no processo de planejamento, assim como na exploração de alguns caminhos latentes, como a sua aplicação na identificação prioridades em subníveis territoriais de análise e no aprofundamento de análises de comorbidades. 


\section{Colaboradores}

M Battesini contribuiu para a concepção, revisão bibliográfica, planejamento, análise e interpretação dos dados. A Fischmann contribuiu para o planejamento, análise e interpretação dos dados. AD Weise trabalhou na redação final do artigo.

\section{Referências}

1. Brasil. Lei no 8.080, de 19 de setembro de 1990. Dispõe sobre as condições para a promoção, proteção e recuperação da saúde, a organização e o funcionamento dos serviços correspondentes e dá outras providências. Diário Oficial da União 1990; 20 set.

2. Brasil. Lei no 8.142 , de 28 de dezembro de 1990 . Dispõe sobre a participação da comunidade na gestão do Sistema Único de Saúde (SUS) e sobre as transferências intergovernamentais de recursos financeiros na área da saúde e dá outras providências. Diário Oficial da União 1990; $31 \mathrm{dez}$.

3. Brasil. Ministério da Saúde (MS). Portaria 3085, de 1 de dezembro de 2006. Regulamenta o Sistema de Planejamento do SUS. Diário Oficial da União 2006; 2 dez.

4. Brasil. Decreto no 7.508, de 28 de junho de 2011. Regulamenta a Lei $n^{\circ} 8.080$, de 19 de setembro de 1990, para dispor sobre a organização do Sistema Único de Saúde - SUS, o planejamento da saúde, a assistência à saúde e a articulação interfederativa, e dá outras providências. Diário Oficial da União 2011; 29 jun.

5. Teixeira CF. Planejamento Municipal em Saúde. Salvador: Cooptec/ISC; 2001.

6. Paim JS. Planejamento em Saúde para não Especialistas. In: Campos GWS, Minayo MCS, Aerkman M, Drumond Júnior M, Carvalho YM. Tratado de Saúde Coletiva. São Paulo: Hucitec; 2006.

7. Bahia L. Afinidades comunicativas e as políticas de saúde no Brasil. Cien Saude Colet 2010; 15(5):22812282.

8. Rivera FJ, Artmann E. Planejamento e gestão em saúde: histórico e tendências com base numa visão comunicativa. Cien Saude Colet 2010; 15(5):22652274.

9. Paim JS. Planejamento e Gestão: para além da comunicação. Cien Saude Colet 2010; 15(5):2279-2280.

10. Patton MQ. Qualitative Research \& Evaluation Methods. Thousand Oaks: Sage; 2002.

11. Santos AR. Metodologia Científica: a construção do conhecimento. Rio de Janeiro; Lamparina; 2007.

12. Monken M, Peiter P, Barcellos C, Iñiguez Rojas L, Navarro MBMA, Gondim GMM, Gracie R. O Território na Saúde: Construindo referências para análises em saúde e ambiente. In: Miranda A, Barcellos C, Moreira JC, Monken M, organizadores. Território, Ambiente e Saúde. Rio de Janeiro: Fiocruz; 2008. p. 23-41

13. Organização das Nações Unidas (ONU). Programa das Nações Unidas para o Desenvolvimento (PNUD). Relatório do Desenvolvimento Humano 2006. A água para lá da escassez: poder, pobreza e a crise mundial da água. New York: PNUD; 2006.

14. Brasil. Ministério da Saúde (MS). Glossário Temático: Sistema de Planejamento, Monitoramento e Avaliação das Ações em Saúde. Brasília: MS; 2006.

15. Olson CL. Statistics: Making sense of data. Boston: Allyn and Bacon; 1987.

16. Coelho TCB, Paim J. Processo decisório e práticas de gestão: dirigindo a Secretaria da Saúde do Estado da Bahia, Brasil. Cad Saude Publica 2005; 21(5): 1373-1382. 
17. Fortes PA. Reflexão bioética sobre a priorização e o racionamento de cuidados de saúde: entre a utilidade social e a eqüidade. Cad Saude Publica 2008; 24(3):696-701.

18. Mattos RA. (Re)visitando alguns elementos do enfoque situacional: um exame crítico de algumas das constribuições de Carlos Mattus. Cien Saude Colet 2010; 15(5):2327-2336.

19. Landerdhal MC, Unfer B, Braun K, Skupien, JA. Resoluções do Conselho de Saúde: instrumento de controle social ou documento burocrático. Cien Saude Colet 2010; 15(5):2431-2436.

20. Cotta RM, Cazal MM, Martins, PC. Conselho municipal de saúde: (re)pensando a lacuna entre o formato institucional e o espaço de participação social. Cien Saude Colet 2010; 15(5):2437-2444.

21. Wendhausen A, Cardoso SM. Processo decisório e Conselhos Gestores de Saúde: aproximações teóricas. Rev Bras Enferm 2007; 60(5):579-584.

Artigo apresentado em 28/08/2012

Aprovado em 02/09/2012

Versão final apresentada em 03/09/2012 